\title{
Brauchen wir ein AYA-Netzwerk?
}

\author{
Jutta Engel ${ }^{a} \quad$ Mathias Freund $^{b}$ Dieter Hölzel ${ }^{a}$ \\ aTumorregister München (TRM), Tumorzentrum München (TZM), \\ ${ }^{\text {b}}$ Klinik für Innere Medizin III, Universitätsklinikum Rostock, Deutschland
}

\section{Epidemiologie von malignen Erkrankungen zwischen 20 und $\mathbf{3 0}$ Jahren \\ Verantwortliche Autoren: Dieter Hölzel, München, Jutta Engel, München}

Basis für epidemiologische Betrachtungen zu Krebserkrankungen sind Tabellen zu alters- und geschlechtsspezifischen Inzidenz- und Mortalitätsraten, die chronologisch und regional untergliedert von Krebsregistern auf nationaler und internationaler Ebene zusammengestellt werden und zum Teil über das Internet offen zugänglich sind [1-3]. Das Risiko, an Krebs zu erkranken, steigt mit dem Lebensalter kontinuierlich an und war im Jahr 2007 in der Altersklasse der 75- bis 80-jährigen Männer mit einer Inzidenz von etwa 2800 je 100000 etwa 22-mal höher als in der Gruppe der 40- bis 45Jährigen (125/100 000). Für das Jahr 2007 schätzte die Gesellschaft der epidemiologischen Krebsregister in Deutschland e.V. (GEKID), dass von den etwa 246000 in Deutschland neu an Krebs erkrankten Männern 77\% im Alter von 50-80 Jahren waren. Bei den Frauen entfielen 63\% der insgesamt 214000 Neuerkrankungen auf diese Altersgruppe.

Dementsprechend gering ist der Anteil junger Menschen im Alter von 20-30 Jahren mit neu diagnostizierter Krebserkrankung: Im Jahr 2007 erhielten etwa 2000 junge Männer und etwa 1800 junge Frauen eine solche Diagnose, während die Krebserkrankungen in der Elterngeneration (60-70 Jahre) bei den Männern um das 37-Fache und bei den Frauen um das 28-Fache höher waren. Im Jahr 2008 standen den etwa 460000 Krebsneuerkrankungen in Deutschland 216000 krebsbedingte Sterbefälle gegenüber, wobei die Krebsmortalität über die vergangenen 2 Dekaden kontinuierlich zurückgegangen ist. Dies ist einerseits den Therapieerfolgen bei fast allen Krebsformen zuzuschreiben, andererseits aber auch einer Verschiebung des Diagnosespektrums: Der Anteil der prognostisch eher ungünstigen Lungen- und Magenkarzinome ist kleiner geworden, während die Fallzahlen an prognostisch günstigen frühen Mamma- und Prostatakarzinomen gestiegen sind. Dies dürfte mit der vermehrten Nutzung von Screening-Maßnahmen zusammenhängen.
Die krebsbedingte Sterberate steigt mit zunehmendem Lebensalter linear an. An Krebs erkrankte Männer im Alter zwischen 20 und 30 Jahren haben eine Überlebenswahrscheinlichkeit von $80 \%$; bei insgesamt niedriger Inzidenz wurden in dieser Altersgruppe im Jahr 2008 insgesamt 257 Krebstodesfälle gemeldet, bei den Frauen waren es 203. Das nach Alter aufgeschlüsselte 10-Jahres-Gesamtüberleben (Abb. 1) beträgt auch bei den im Tumorregister München dokumentierten Patienten in den 3 jüngeren Altersklassen ( $<20$ Jahre, 20-29 Jahre, 30-39 Jahre) etwa $80 \%$.

Krebskranke junge Frauen haben eine ähnliche Überlebenswahrscheinlichkeit wie die altersgleichen Männer; dies gilt allerdings nur bis zu einem Alter von 30 Jahren, danach wirkt sich der hohe Anteil an Mammakarzinomen nachteilig aus. Dies zeigt auch ein Vergleich der häufigsten Neuerkrankungen bei Männern (Tab. 1) und Frauen (Tab. 2): Bei jungen Männern handelt es sich bei $45 \%$ der Krebsneuerkrankungen um Hodentumoren, die mit etwa 97\% Heilungen eine sehr günstige Prognose haben. Bei jungen Frauen steht an erster Stelle der Krebsdiagnosen das Melanom, gefolgt von Brustkrebs. Auch im jungen Lebensalter ist ein breites Spektrum an Krebserkrankungen vorhanden bei insgesamt jedoch kleineren Fallzahlen. Die Patienten werden in der Regel qualifizierten onkologischen Zentren zugewiesen, wobei die Heterogenität der Erkrankungen bei kleinen Patientenzahlen und die vielen Zentren keine relevanten Aussagen zur Qualität der Versorgung in den Zentren erlauben.

Nicht zu vernachlässigen ist die Inzidenz von Zweitmalignomen bei Patienten, die zum Zeitpunkt der ersten Krebsdiagnose noch relativ jung waren. Es ist davon auszugehen, dass fast $10 \%$ der 30-jährigen Patienten innerhalb von 10 Jahren ein Zweitmalignom entwickelt. Daraus leitet sich ein erhöhter Beratungsbedarf für junge Krebspatienten im Rahmen der Nachsorge ab. Derzeit leben in Deutschland etwa 17000 Menschen, die im Alter von 20-30 Jahren erstmals an Krebs erkrankt waren. Unter Hinzunahme der Patienten mit Krebs im Kindes- und Jugendalter ergibt sich eine Prävalenz von 37000 Krebspatienten in der Gruppe der jetzt 20- bis 30-Jährigen. Der überwiegende Teil dieser Patienten hat die Krebserkrankung überwunden und

\section{KARGER}

Fax +497614520714

Information@Karger.de

www.karger.com (c) 2011 S. Karger GmbH, Freiburg

0378-584X/11/3417-0002\$38.00/0

Accessible online at:

www.karger.com/onk 


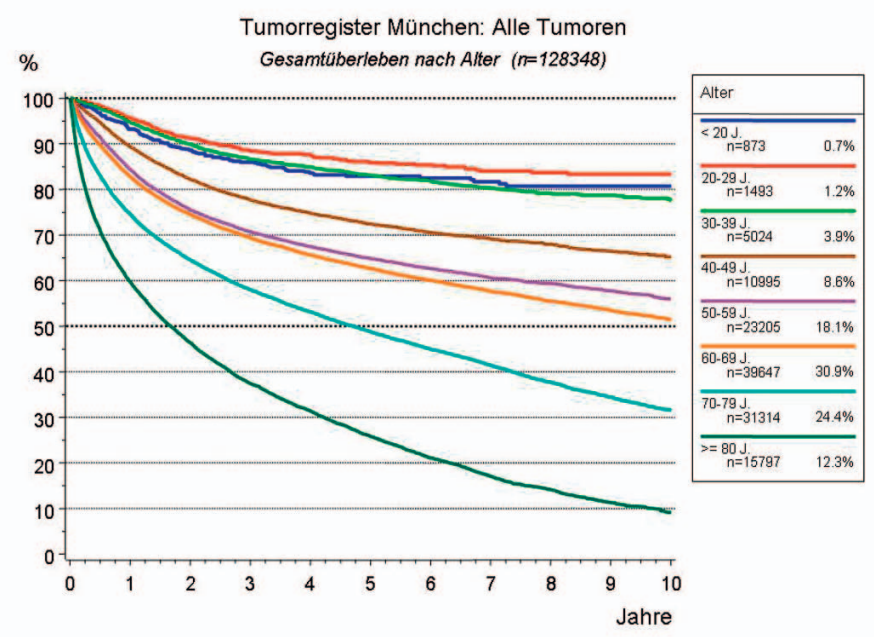

Abb. 1. Gesamtüberleben und relatives Überleben nach einer Krebserkrankung in Abhängigkeit vom Alter. Das relative Überleben ist eine Schätzung für das tumorspezifische Überleben, das sich durch Bezug auf das Überleben der Normalbevölkerung ergibt. Damit ergeben sich deutliche Unterschiede in den hohen Alterklassen. Insgesamt wird aus dem relativen Überleben das Verhältnis von 216000 krebsbedingten Sterbefällen und 460000 Neuerkrankungen ersichtlich (53\% Überleben).

Tab. 1. Verteilung der Krebserkrankungen nach der Häufigkeit der Erkrankungen in 10-Jahres-Altersklassen für Männer. Die Zahlen basieren auf den Schätzungen von GEKID. Die Zeile «Rest» gibt den Anteil aller nicht genannten Diagnosen an, die Zeile «Syst» den Anteil aller systemischen Erkrankungen in der jeweiligen Altersklasse

\begin{tabular}{|c|l|c|c|c|c|c|c|c|}
\hline \multicolumn{7}{|c|}{ häufige Neuerkrankungen (Männer) } \\
\hline & \multicolumn{2}{|c|}{$20-30 \mathrm{~J}$} & \multicolumn{2}{c|}{$30-40 \mathrm{~J}$} & \multicolumn{2}{c|}{$40-50 \mathrm{~J}$} & \multicolumn{2}{c|}{$50-60 \mathrm{~J}$} \\
\hline Rang & Diagnose & $\%$ & Diagnose & $\%$ & Diagnose & $\%$ & Diagnose & $\%$ \\
\hline 1 & Hoden & 45 & Hoden & 33 & Lunge & 11 & Prostata & 20 \\
\hline 2 & M.Hodgkin & 10 & Melanom & 11 & Kopf-Hals & 11 & Lunge & 14 \\
\hline 3 & Melanom & 9 & Darm & 13 & Darm & 11 & Darm & 14 \\
\hline 4 & ZNS & 6 & Schilddr. & 7 & Hoden & 9 & Kopf-Hals & 9 \\
\hline 5 & NHL & 5 & NHL & 7 & Melanom & 7 & Harnblase & 6 \\
\hline Rest & & 25 & & 29 & & 51 & & 37 \\
\hline Syst. & & 20 & & 15 & & 10 & & 6 \\
\hline
\end{tabular}

Tab. 2. Verteilung der Krebserkrankungen nach der Häufigkeit der Erkrankungen in 10-Jahres-Altersklassen für Frauen (siehe Legende Tab. 1)

\begin{tabular}{|c|c|c|c|c|c|c|c|c|}
\hline \multicolumn{9}{|c|}{ häufige Neuerkrankungen (Frauen) } \\
\hline & \multicolumn{2}{|c|}{$20-30 \mathrm{~J}$} & \multicolumn{2}{|c|}{$30-40 \mathrm{~J}$} & \multicolumn{2}{|c|}{$40-50 \mathrm{~J}$} & \multicolumn{2}{|c|}{$50-60 \mathrm{~J}$} \\
\hline Rang & Diagnose & $\%$ & Diagnose & $\%$ & Diagnose & $\%$ & Diagnose & $\%$ \\
\hline 1 & Melanom & 23 & Brust & 35 & Brust & 47 & Brust & 41 \\
\hline 2 & Brust & 13 & Melanom & 13 & Melanom & 6 & Darm & 10 \\
\hline 3 & Schilddr. & 12 & Gebärm.H & 12 & Gebärm.H & 6 & Lunge & 8 \\
\hline 4 & M.Hodgkin & 10 & Schilddr. & 9 & Darm & 6 & Gebärm.H & 6 \\
\hline 5 & Gebärm.H & 9 & Darm & 4 & Lunge & 5 & Ovar & 4 \\
\hline Rest & & 33 & & 27 & & 30 & & 31 \\
\hline Syst. & & 20 & & 7 & & 4 & & 5 \\
\hline
\end{tabular}

Brauchen wir ein AYA-Netzwerk? befindet sich in der Nachsorge. Dies stellt die eigentliche Herausforderung für die Versorgungssysteme dar, die insbesondere hinreichend Beratungen für diese geheilten Patienten mit ihren Fragen bezüglich der Lebens- und Familienplanung anbieten sollten.

Fazit

Krebserkrankungen im jüngeren Lebensalter treten in vielfältiger Form auf und sind eine Herausforderung für alle medizinischen Fachgebiete. Die Gesamtzahl der jährlich auftretenden Neuerkrankungen ist mit etwa 3850 Patienten (20- bis 30 -Jährige) oder $0,84 \%$ relativ klein im Vergleich zur Gesamtzahl der Erkrankungen. Bei längerfristigem Follow-up ergibt sich dennoch für das Altersintervall der 20bis 30-Jährigen eine Prävalenz von 37000 Patienten, die irgendwann in ihrem jungen Leben an Krebs erkrankt sind. Wird auch die Altersklasse der bis zu 40-Jährigen in die Gruppe der «Adolescents and Young Adults» (AYA) mit einbezogen, summiert sich die Zahl der Patienten mit einer aktuellen oder zurückliegenden Krebserkrankung auf insgesamt 95000 Betroffene. Speziell für die Krebserkrankungen junger Menschen zugeschnittene Krebsregister sind nicht zu begründen, da im internationalen Vergleich keine Defizite in der Therapie zu bestehen scheinen und viele Leistungen wie in einem Krebsregister für Erwachsene deshalb doppelt und ineffizient zu erbringen wären. Umso wichtiger ist eine qualitativ hochwertige onkologische Versorgung für alle Krebspatienten sowie die Etablierung regionaler klinischer Krebsregister, die an die Stelle von Spartenaktivitäten und wenig effektiven Beratungsangeboten treten sollten. Ein von der Onkologie gemeinsam getragenes Register-Konzept sowie dessen Umsetzung sind voranzutreiben, wobei jedes Fachgebiet die verfügbaren Daten permanent diskutieren sollte. In den Auseinandersetzungen um Qualitätssicherung und explodierende Kosten im Gesundheitswesen ist die Unterfütterung mit Daten aus der onkologischen Routineversorgung unerlässlich. Der Verzicht auf solche evidenzbasierten Aussagen zur Versorgungsqualität würde lediglich zum Aufbau weiterer bürokratischer Kontrollsysteme führen.

\section{Brauchen wir ein AYA-Netzwerk? \\ Verantwortlicher Autor: Mathias Freund, Rostock}

Die Idee, für Krebspatienten im Jugendalter spezielle Versorgungsangebote auszuarbeiten, ist nicht neu. So hat das University of Pittsburgh Cancer Institute (UPCI) seit längerem ein Onkologie-Programm für AYA etabliert, mit dem junge Krebspatienten im Alter von 15-21 Jahren der jeweils optimalen Therapieschiene (Pädiatrie, Erwachsenen-Onkologie) zugeordnet werden und die auch eine den Bedürfnissen ihres Alters entsprechende psychosoziale Unterstützung bekommen. Auch hierzulande wird die Frage der Versorgung der Heranwachsenden und jungen Erwachsenen sehr kontrovers 
diskutiert. Es muss realistisch gesehen werden, dass die Diskussion durch berufspolitische Interessen stark beeinflusst wird. Das Problem kann aber nicht einfach auf die Frage reduziert werden, ob sich ein solcher Patient eher an eine pädiatrische Einrichtung wenden sollte oder ob er in der Erwachsenen-Onkologie besser aufgehoben ist. Ein solches Herangehen greift zu kurz.

Wir müssen berücksichtigen, dass die AYA-Krebsproblematik vielschichtig ist. $\mathrm{Zu}$ bedenken sind mögliche Unterschiede in der Biologie der Krebserkrankungen ebenso wie pubertätsbedingte hormonelle Einflüsse auf das Krankheitsgeschehen und deren Effekte auf die Pharmakokinetik und den Metabolismus von Krebsmedikamenten. Diese Zusammenhänge sind sicherlich noch näher zu untersuchen. Hinzu kommen die psychosozialen Rahmenbedingungen, die diese Gruppe von Krebspatienten auszeichnet. Dies betrifft sowohl Besonderheiten in der Krankheitsakzeptanz und -verarbeitung als auch eine gewisse Instabilität und Neuorientierung der Persönlichkeit. Die Compliance für eine Therapie über längere Zeiträume ist nicht immer gegeben, weil die Jugendlichen mit der Lösung vom Elternhaus und dem Berufseinstieg andere Prioritäten setzen. Welche Gruppen von Patienten letztlich den AYA zuzuordnen sind - die Jugendlichen von 15-20 oder auch die jungen Erwachsenen bis zum Alter von 30 oder gar 35 Jahren -, bleibt vorerst offen.

Die altersbedingte psychosoziale Problematik findet sich im Übrigen nicht nur bei Krebspatienten, sondern sollte bei allen jungen Menschen mit chronischen Krankheiten bedacht werden, etwa bei Post-Transplantationspatienten und Diabetikern. Für die medizinische Versorgung ergibt sich daraus die Notwendigkeit, in verschiedenen Fachbereichen miteinander zu kooperieren - das kann weit über die rein onkologische Versorgung hinaus reichen. Auch hier bietet ein AYA-Netzwerk die Möglichkeit, den interdisziplinären Anspruch an die Versorgung von AYA-Patienten umzusetzen.

Aus diesen Überlegungen heraus wurde 2010 am Universitätsklinikum Rostock eine AYA-Arbeitsgruppe etabliert, der unter anderem Pädiater, Gynäkologen, internistisch und hämatologisch ausgerichtete Onkologen sowie Psychologen angehören. Mit welchen Patienten wir es zu tun haben, lässt sich aus dem klinischen Krebsregister Mecklenburg-Vorpommern entnehmen. Es erfasst vollständig die Krebserkrankungen in den Einzugsgebieten Greifswald, Neubrandenburg, Rostock und Schwerin. In den Jahren 2000-2009 erkrankten 2164 Einwohner im Alter von 15-40 Jahren neu an Krebs [4], wobei Frauen häufiger betroffen waren als Männer. Die Zuordnung der Tumoren erfolgte nach der Internationalen Klassifikation der Krankheiten (ICD)-10 in die Obergruppen der bösartigen Neubildungen. Der Anteil von Tumoren des lymphatischen und blutbildenden Systems mag mit 13,6\% (Tab. 3) recht gering erscheinen, werden doch diese Tumoren häufig als «klassische» Krebserkrankungen im jungen Erwachsenenalter angesehen.
Tab. 3. AYA: Zahlen aus dem Krebsregister Rostock (nach [4])

\begin{tabular}{|c|c|c|c|}
\hline Atmungsorgane & 43 & $3,1 \%$ & \multirow{14}{*}{$\begin{array}{r}1402 \\
\text { Fälle } \\
\text { ca. } 3 \% \\
\text { aller } \\
\text { Tumore }\end{array}$} \\
\hline Schilddrüse & 160 & $11,4 \%$ & \\
\hline Auge, Gehim, sonst. ZNS & 39 & $2,8 \%$ & \\
\hline Brustdrüse & 184 & $13,1 \%$ & \\
\hline Lippe, Mundhöhle, Pharynx & 35 & $2,5 \%$ & \\
\hline Verdauungsorgane & 101 & $7,2 \%$ & \\
\hline Lymphatisch, blutbildend & 191 & $13,6 \%$ & \\
\hline Idiopathische Aplastische Anämie & 1 & $0,1 \%$ & \\
\hline Melanom & 268 & $19,1 \%$ & \\
\hline Mesotheliales Gewebe & 21 & $1,5 \%$ & \\
\hline Knochen & 5 & $0,4 \%$ & \\
\hline Harnorgane & 41 & $2,9 \%$ & \\
\hline Männliche Genitalorgane & 163 & $11,6 \%$ & \\
\hline Weibliche Genitalorgane & 150 & $10,7 \%$ & \\
\hline Gutartige Neubildungen & 2 & & \\
\hline In-situ-Neubildungen & 705 & & \\
\hline nicht näher bez. Lokalisation & 18 & & \\
\hline Unbekanntes Verhalten & 37 & & \\
\hline Gesamt & 2164 & Fälle & \\
\hline
\end{tabular}

Tatsächlich waren jedoch Melanome (in der Regel nicht metastasierte Tumoren) in der Altersgruppe der unter 40-Jährigen häufiger als Leukämien und Lymphome, des Weiteren hatten Karzinome der Brustdrüse, der Schilddrüse und der Genitalorgane höhere Anteile an den Gesamterkrankungen. Das Spektrum der Krebserkrankungen ändert sich mit dem Alter, wobei die Zahl hämatologischer Neoplasien sinkt und Sarkome an Bedeutung gewinnen.

Einen enorm hohen Anteil an den Krebsneuerkrankungen der über 20-jährigen Frauen haben Mamma- und Zervixkarzinome (Letztere zumeist als Carcinoma in situ). Dieses heterogene Spektrum an Erkrankungen erfordert eine differenzierte Betrachtungsweise und sehr unterschiedliche Therapiestrategien. Jedoch werden längst nicht alle jungen Patienten in onkologischen Zentren behandelt, so etwa wird im ländlichen Mecklenburg-Vorpommern ein relativ großer Teil von ihnen auch in kleineren Kliniken und von niedergelassenen Ärzten medizinisch versorgt.

\section{Fazit}

Die AYA machen etwa 3\% der Tumorerkrankten aus und sind damit eine relativ kleine Patientengruppe. Dennoch verlangt diese Altersgruppe besondere Aufmerksamkeit, zumal viele dieser Patienten kurativ behandelt werden können. Nebenwirkungen von Krebstherapien sind für AYA von großer Bedeutung, denn aufgrund ihrer noch langen Lebenserwartung stellen sich explizit Fragen zum Umgang mit den Spätfolgen bzw. Langzeitnebenwirkungen der verschiedenen Therapieformen. Erschwert wird die Betreuung dieser Patienten dadurch, dass bei ihnen ein großes Spektrum an Neoplasien auftreten kann und sich allenfalls an Zentren eine grö- 
Bere Zahl an Patienten mit ähnlichem Erkrankungsbild zusammenfindet. Häufig werden die Patienten dezentral betreut und pendeln zwischen ambulanter und stationärer Versorgung.

Um den Bedürfnissen der AYA besser gerecht zu werden, ist ein interdisziplinärer Ansatz unter Einbeziehung von Pädiatern, Erwachsenen-Internisten/Onkologen, Psychologen, Psychoonkologen und Sozialarbeitern nötig. Ferner sollte eine stärkere Anbindung an Grundlagenforschung und klinische Studien angestrebt werden. Einem sektorenübergreifenden Ansatz folgend möchte das Netzwerk möglichst viele Einrichtungen einbinden. Eine Begrenzung auf den stationären Bereich, z.B. im Sinne der Einrichtung einer AYA-Station wäre nicht sinnvoll, da der Wechsel zwischen stationären Aufenthalten und ambulanter Betreuung grade bei diesen Patienten charakteristisch ist. Nicht zuletzt sollten auch Projekte ins Auge gefasst werden, die auf die Langfristperspektive der Patienten ausgerichtet sind: Hier geht es um das Management von Nebenwirkungen, um Rehabilitation und die Umsetzung sozialtherapeutischer Konzepte bis hin zu Erlebnis- und Eventtherapie.
Hilfreich könnte die Bildung von regionalen AYA-Kompetenzteams sein, die eine transsektorale und langfristige Betreuung anbieten. Sie können die Koordination von Diagnostik und Behandlung übernehmen sowie eigene, auf ihre Patienten zugeschnittene Angebote machen. Wir sollten die Bildung von AYA-Teams als Herausforderung annehmen. Die Versorgung dieser besonderen Gruppe von Patienten erfordert einen ganzheitlichen Therapieansatz und eine verstärkte multidisziplinäre Zusammenarbeit. Die Deutsche Gesellschaft für Hämatologie und Onkologie e.V. (DGHO) sieht in der Etablierung eines AYA-Netzwerkes eine große Chance, die Situation junger Krebspatienten zu verbessern, und lädt dazu ein, sich an der Arbeit aktiv zu beteiligen.

\section{Disclosure Statement}

Es bestehen keine Interessenkonflikte.

\section{Literatur \\ www.tumorregister-muenchen.de. \\ www.gekid.de. \\ 3 www.globocan.iarc.fr.}

4 Hilgendorf I et al.: Onkologische Erkrankungen bei Jugendlichen und jungen Erwachsenen Zahlen aus dem Klinischen Krebsregister Rostock als Arbeitsgrundlage für eine interdisziplinäre Herausforderung. Tumordiagnostik Therapie 2011; 32:85-92. 